\title{
Progress Towards Integrated Photon-Pair Sources and Their Applications (1640968-Y5)
}

\section{SHAYAN MOOKHERJEA}

Department of Electrical and Computer Engineering, University of California, San Diego, MC 0407 La Jolla CA 92093-0407 USA

Email:smookher@ucsd.edu

Summary of a Project Outcomes report of research funded by the U.S. National Science Foundation under Project Number 1640968 (Year 5).

\section{Introduction and Goals}

The objective of this project was to make significant advances in quantum optical communications through the design, fabrication and demonstration of novel devices at the microchip scale. The principal goal of the UCSD subproject was to develop key building blocks for photonic microchips that are energy-efficient, leverages modern micro-fabrication platforms, reduces operational complexity and improve scalability with the potential for future adoption by industry.

\section{Activities}

Figure 1 shows a snapshot of the major outcomes of research and educational activities performed across the three universities participating in this project.

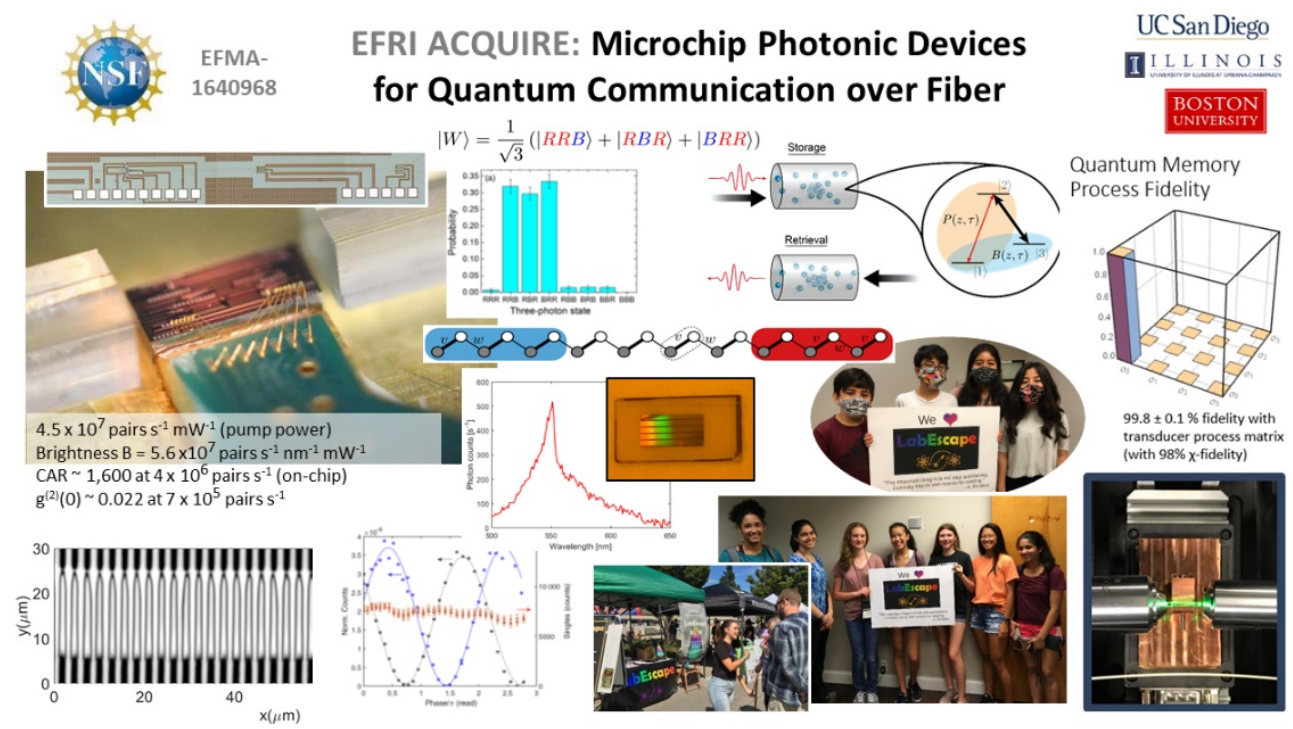

Figure 1 Snapshot of outcomes of the multi-site research and educational project.

The main research outcomes of the UCSD sub-project include the design and demonstration of entangled photon-pair generation with record performance in both silicon photonics and thin-film lithium niobate. In particular, we made significant improvements in our pair generation devices using high-Q silicon 
microring resonators. Prior to this project, we have studied pair generation using the silicon coupled-resonator optical waveguide structure[1]-[4], which has a resonantly enhanced four-wave mixing nonlinearity but uses lower quality factor resonators coupled in a serial cascade [5]. We have demonstrated the time-energy entangled nature of the photon pairs generated by our devices around $1550 \mathrm{~nm}[6]$. However, such devices are sensitive to fabrication disorder and in practice, an optimized single microring resonator may achieve better performance. Among the reasons for improved overall performance are the improvements we have made in monitoring and stabilizing the pair generation process[7] which help in aligning the pump wavelength to the high-Q resonance.

Our research activities include: measurements of silicon photonic entangledpair and heralded single photon generation using an external laser diode, using a hybrid silicon laser and using a fraction of a PRBS-modulated data carrying sequence of optical pulses as the pump for the spontaneous four-wave mixing process.

Devices were designed and fabricated at Sandia National Labs using a multiproject wafer process for silicon photonics [8].

Table 1 shows a comparison between our silicon photonic microring device (SFWM source of entangled photons) and a TFLN waveguide, both of which were studied in this project. The latter achieves the same low-noise performance that silicon is known for (in contrast to a silica source of entangled photons), but also benefits from nearly one order of magnitude improvement in the figure-of-merit CAR x PGR, i.e., the same signal-to-noise ratio can be achieved at one order of magnitude higher pair generation rates. With only modest improvements (e.g., a factor of 2 increased length or slightly better phase matching), these integrated devices should outperform the best SPDC sources realized using bulk crystals and much higher pump powers

\begin{tabular}{|c|c|c|c|}
\hline $\begin{array}{l}\text { Core material } \\
\text { (core thickness) }\end{array}$ & $\begin{array}{l}\text { Si } \\
(220 \mathrm{~nm})\end{array}$ & $\begin{array}{l}\text { (thin-film) } \\
(300 \mathrm{~nm})\end{array}$ & $\mathbf{L N}$ \\
\hline nonlinearity & $\begin{array}{l}\text { spontaneous four-wave mixing } \\
\text { (SFWM) }\end{array}$ & $\begin{array}{l}\text { spontaneous parametric } \\
\text { conversion (SPDC) }\end{array}$ & down- \\
\hline type & Micro-resonator & Waveguide with $\mathrm{QPM}^{(\mathrm{a})}$ & \\
\hline bandwidth & $1.4 \mathrm{GHz}$ & $140 \mathrm{GHz}$ & \\
\hline footprint & $20 \mathrm{~mm} \times 20 \mathrm{~mm}$ & $5 \mathrm{~mm} \times 2 \mathrm{~mm}$ & \\
\hline CAR x PGR ${ }^{(b)}$ & $\begin{array}{l}0.6 \quad \mathrm{x} \quad 10^{9} \quad \text { pairs. }^{-1} \\
\text { CAR 532 at } 1.1 \times 10^{6} \text { pairs.s }{ }^{-1}\end{array}$ & $\begin{array}{lcc}7.6 & \mathrm{x} & 10^{9} \\
\text { CAR } & 668 \text { at } & 1.1 \times 10^{7} \text { pairs.s }\end{array}$ & pairs.s ${ }^{-1}$ \\
\hline $\mathrm{g}^{(2)} \mathrm{H}(0)^{(\mathrm{c})}$ & $\begin{array}{l}0.0053 \quad \pm \\
\text { at } \mathrm{N}_{\mathrm{H}}=18 \text { kilo-pairs/s }\end{array}$ & $\begin{array}{l}0.0022 \quad \pm \\
\text { at } \mathrm{N}_{\mathrm{H}}=15 \text { kilo-pairs/s }\end{array}$ & 0.0004 \\
\hline $\begin{array}{l}\text { Heralding (Klyshko) } \\
\text { efficiency }^{(\mathrm{d})}\end{array}$ & $4 \%$ & $2 \%$ & \\
\hline
\end{tabular}


(b) CAR: Coincidences-to-accidentals ratio; PGR: Pair-generation rate. Generally, at high PGR, CAR $\propto(\mathrm{PGR})^{-1}$.

(c) heralded self-autocorrelation at zero time delay; $\mathrm{N}_{\mathrm{H}}=$ (off-chip) detected herald rate.

(d) Including all on-chip and off-chip loss contributions.

\section{Impact}

Silicon photonics is a scalable and manufacturable device technology. The realization of high-performance pair-source devices using this platform may enable lower cost and greater scalability of entangled photon pair generation devices, and lead to greater deployment in practical systems.

Lithium niobate waveguides require periodic poling in order to achieve quasiphase matching and efficient conversion from a pump at $780 \mathrm{~nm}$ to entangled photons at $1560 \mathrm{~nm}$. Short-period poling methods for quasi-phase matching in nonlinear waveguides were developed, with periods less than 1 micron, and studied using a variety of methods including nonlinear microscopy techniques.

A transceiver that is suitable for quantum communications also requires an efficient and low-noise single-photon detector. Modern superconducting nanowire single-photon detectors (SPSNDs) offer the best performance, and can achieve a timing jitter of less than 40 ps (in some cases, less than $10 \mathrm{ps}$ ), and although such detectors need cryogenic cooling, fridge technology is becoming more practical. The lithium niobate platform is also interesting because SNSPDs can be integrated on this platform, and the low pump powers and high efficiency make optical filtering easier. While the complete realization of an integrated transceiver is beyond the scope of this project, it is important at this stage to optimize the pump coupling and SPDC stages of the device, and study the design of pump-separation filters, towards the eventual goal of integration.

Low-jitter superconducting nanowire single-photon detectors were used to improve waveform capture techniques, extending the state-of-the-art in highbandwidth sampling oscilloscope methods by nearly a factor of 10 in bandwidth and also improving the sensitivity of the measurement down to the picowatt level, about seven orders of magnitude lower than a standard sampling oscilloscope. A potential application of these techniques is to help manufacturing of integrated photonics devices, and lessen the burden on packaging and testing, which are currently among the most expensive and timeconsuming steps.

One of the advantages of the integrated device is that the coupling to the resonator is well controlled, although the dispersion (i.e., wavelength sensitivity) of the directional coupler can be large. However, the same large dispersion issue also affects the coupled-resonator device and can sometimes be used for added functionality[9], [10]. 
The work of three graduate students at UCSD was supported by this project. They received hands-on training in the fields of electrical engineering, physics, device fabrication and measurement and materials science. Results have been published in peer-reviewed journals and presented at internationallyrecognized conferences, as well as workshops, seminars, and meetings. Students and researchers from the different groups met and exchanged ideas at major conferences such as CLEO.

\section{Potential Subsequent Extensions}

Scalable quantum networking will be dependent on compact, manufacturable devices which provides the necessary attributes for distributed communications at the single-photon level. In a network, multi-photon operations such as entanglement swapping and quantum relay are performed at several nodes. Existing systems typically implement these operations by performing a Bell state measurement on a two-photon input. Teleportation, also known as a quantum relay, uses the physical process of measurement to transfer quantum information from an incoming photon to an outgoing one, and thus overcomes the distance limitations of direct quantum communications. An entangled photon generator comprises the "EPR" (Einstein-Podolsky-Rosen) state source, which provides the photon pairs needed for entanglement swapping and teleportation.

Among various techniques of making a source of entangled photons, the method of generating entangled photons using a spontaneous optical nonlinearity in a nonlinear optical device stands out. These devices have demonstrated room temperature operation, record brightness (proportional to the number of photons per second), and superior figures-of-merit.

Spontaneous parametric down-conversion (SPDC) was used in the paradigmatic demonstrations of teleportation, entanglement-based cryptography, tests of Bell's inequality, one-way optical quantum computing and other notable experiments. SPDC sources of entangled photon pairs are used in the Chinese "Micius" satellite and the Singapore "SpooQy-1" satellite.

SPDC or spontaneous four-wave mixing (SFWM) sources are also used for demonstrating scalable quantum information processing at the microchip level. Today, entangled photon-pair source devices are available from both research and commercial sources, similar to crystals for other nonlinear optics applications such as second-harmonic generation and wavelength conversion. Many traditional designs do not generally benefit from modern silicon-based microfabrication, which can realize hundreds or thousands of devices on a wafer, and create complex integrated circuits.

While it is possible to induce a weak $\chi(2)$ effect in centrosymmetric materials such as silicon and silicon nitride, it is also possible to bring the stronger $\chi(2)$ effect of lithium niobate (LN) into a manufacturable integrated photonics 
ecosystem, for example, by using thin-film LN and bonding technology. Because of the small waveguide cross-sectional area, significant performance benefits are also achieved. These waveguides have a higher group-velocity dispersion than traditional LN waveguides, and require a shorter quasi-phase matching (QPM) poling period, but also achieve a narrower phase matching spectral bandwidth (about $1 \mathrm{~nm}$ ). In contrast, the wide QPM bandwidth (tens of nm) of traditional SPDC requires ultrashort optical pump pulses generated by a mode-locked laser, which is difficult to integrate monolithically. Furthermore, for time-frequency mode processing, sub- $\mathrm{THz}$ bandwidths are easier to handle with integrated electro-optic modulators (EOMs).

Our first demonstration of a hybrid silicon laser pumping silicon photonic entangled-pair generation and heralded single-photon generation was published as a post-deadline paper at CLEO. This study may be useful for developing a practical roadmap towards silicon-photonics foundry (wafer) scale fabrication of the laser that is typically required for entangled photon state generation. However, one challenge is that short pump pulses are usually used for pair generation (from mode locked lasers), and although integrated electro-optic modulators can be used to carve pump pulses, they may need a very wide bandwidth that is beyond the capabilities of traditional silicon photonics[11].

One of the challenges of high index contrast integrated photonics using microresonators is the sensitivity to disorder, as we have modeled in the coupled-resonator configuration[12], [13]. However, we have also studied ways to study and potentially compensate for disorder after fabrication [14] [16].

These devices use the efficient all-optical four-wave mixing (FWM) nonlinearity that occurs in a highly confined waveguide (cross sectional mode area about 0.2 squared microns). Physics theory suggests that there are other interesting Kerr nonlinear effects that might occur in appropriately dispersionengineered devices, such as ways to prevent the spreading of light through soliton generation [17]. These could be alternative ways to shape the pump beam.

\section{Open-Access Reporting Initiative}

PRAISE: This open-access document is provided in support of our PRAISE

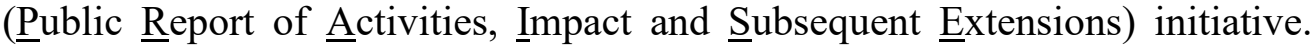
What is it? An open-access document shared with the public which describes the research outcomes of publicly-funded projects such as those funded by the U.S. NSF (National Science Foundation).

\section{References}


[1] S. Mookherjea, "Spectral characteristics of coupled resonators," J. Opt. Soc. Am. B, vol. 23, no. 6, p. 1137, Jun. 2006, doi: 10.1364/JOSAB.23.001137.

[2] S. Mookherjea, D. S. Cohen, and A. Yariv, "Nonlinear dispersion in a coupled-resonator optical waveguide," Opt. Lett., vol. 27, no. 11, p. 933, Jun. 2002, doi: 10.1364/OL.27.000933.

[3] J. R. Ong and S. Mookherjea, "Quantum light generation on a silicon chip using waveguides and resonators," Opt. Express, vol. 21, no. 4, p. 5171, Feb. 2013, doi: 10.1364/OE.21.005171.

[4] M. L. Cooper et al., "235-ring Coupled-Resonator Optical Waveguides," in Conference on Lasers and Electro-Optics 2010, San Jose, California, 2010, p. CTuHH3. doi: 10.1364/CLEO.2010.CTuHH3.

[5] J. R. Ong, R. Kumar, and S. Mookherjea, "Silicon microring-based wavelength converter with integrated pump and signal suppression," Opt. Lett., vol. 39, no. 15, p. 4439, Aug. 2014, doi: 10.1364/OL.39.004439.

[6] R. Kumar, M. Savanier, J. R. Ong, and S. Mookherjea, "Entanglement measurement of a coupled silicon microring photon pair source," Opt. Express, vol. 23, no. 15, p. 19318, Jul. 2015, doi: 10.1364/OE.23.019318.

[7] M. Savanier, R. Kumar, and S. Mookherjea, "Optimizing photon-pair generation electronically using a $p-i-n$ diode incorporated in a silicon microring resonator," Appl. Phys. Lett., vol. 107, no. 13, p. 131101, Sep. 2015, doi: 10.1063/1.4932047.

[8] A. L. Lentine et al., "Silicon photonics platform for national security applications," in 2015 IEEE Aerospace Conference, Big Sky, MT, Mar. 2015, pp. 1-9. doi: 10.1109/AERO.2015.7119249.

[9] S. Mookherjea, "Using gain to tune the dispersion relation of coupledresonator optical waveguides," IEEE Photon. Technol. Lett., vol. 18, no. 5, pp. 715-717, Mar. 2006, doi: 10.1109/LPT.2006.871144.

[10] S. Mookherjea, "Semiconductor coupled-resonator optical waveguide laser," Appl. Phys. Lett., vol. 84, no. 17, pp. 3265-3267, Apr. 2004, doi: 10.1063/1.1719278.

[11] X. Wang, P. O. Weigel, J. Zhao, M. Ruesing, and S. Mookherjea, "Achieving beyond-100-GHz large-signal modulation bandwidth in hybrid silicon photonics Mach Zehnder modulators using thin film lithium niobate," APL Photonics, vol. 4, no. 9, p. 096101, Sep. 2019, doi: $10.1063 / 1.5115243$.

[12] M. L. Cooper and S. Mookherjea, "Modeling of Multiband Transmission in Long Silicon Coupled-Resonator Optical Waveguides," IEEE Photon. Technol. Lett., vol. 23, no. 13, pp. 872-874, Jul. 2011, doi: 10.1109/LPT.2011.2141657.

[13] S. Mookherjea and M. A. Schneider, "Avoiding bandwidth collapse in long chains of coupled optical microresonators," Opt. Lett., vol. 36, no. 23, p. 4557, Dec. 2011, doi: 10.1364/OL.36.004557. 
[14] M. L. Cooper, G. Gupta, J. S. Park, M. A. Schneider, I. B. Divliansky, and S. Mookherjea, "Quantitative infrared imaging of silicon-on-insulator microring resonators," Opt. Lett., vol. 35, no. 5, p. 784, Mar. 2010, doi: 10.1364/OL.35.000784.

[15] S. Mookherjea and H. R. Grant, "High dynamic range microscope infrared imaging of silicon nanophotonic devices," Opt. Lett., vol. 37, no. 22, p. 4705, Nov. 2012, doi: 10.1364/OL.37.004705.

[16] Y. Shen, I. B. Divliansky, D. N. Basov, and S. Mookherjea, "Perfect set-and-forget alignment of silicon photonic resonators and interferometers," in Optical Fiber Communication Conference/National Fiber Optic Engineers Conference 2011, Los Angeles, California, 2011, p. PDPC3. doi: 10.1364/OFC.2011.PDPC3.

[17] B. Crosignani, A. Yariv, and S. Mookherjea, "Nonparaxial spatial solitons and propagation-invariant pattern solutions in optical Kerr media," Opt. Lett., vol. 29, no. 11, p. 1254, Jun. 2004, doi: 10.1364/OL.29.001254. 